\title{
Collaborative Training With a More Experienced Partner: Remediating Low Pretraining Self-Efficacy in Complex Skill Acquisition
}

\author{
Eric Anthony Day, Paul R. Boatman, Vanessa Kowollik, Jazmine Espejo, Lauren E. \\ McEntire, and Rachel E. Sherwin, University of Oklahoma, Norman, Oklahoma
}

\begin{abstract}
Objective: This study examined the effectiveness of collaborative training for individuals with low pretraining self-efficacy versus individuals with high pretraining selfefficacy regarding the acquisition of a complex skill that involved strong cognitive and psychomotor demands. Background: Despite support for collaborative learning from the educational literature and the similarities between collaborative learning and interventions designed to remediate low self-efficacy, no research has addressed how selfefficacy and collaborative learning interact in contexts concerning complex skills and human-machine interactions. Method: One hundred fifty-five young male adults trained either individually or collaboratively with a more experienced partner on a complex computer task that simulated the demands of a dynamic aviation environment. Participants also completed a task-specific measure of self-efficacy before, during, and after training. Results: Collaborative training enhanced skill acquisition significantly more for individuals with low pretraining self-efficacy than for individuals with high pretraining self-efficacy. However, collaborative training did not bring the skill acquisition levels of those persons with low pretraining self-efficacy to the levels found for persons with high pretraining self-efficacy. Moreover, tests of mediation suggested that collaborative training may have enhanced appropriate skill development strategies without actually raising self-efficacy. Conclusion: Although collaborative training can facilitate the skill acquisition process for trainees with low self-efficacy, future research is needed that examines how the negative effects of low pretraining self-efficacy on complex skill acquisition can be more fully remediated. Application: The differential effects of collaborative training as a function of self-efficacy highlight the importance of person analysis and tailoring training to meet differing trainee needs.
\end{abstract}

\section{INTRODUCTION}

Self-efficacy, which refers to a person's belief that he or she has the capacity to successfully perform specific behaviors or tasks (Bandura, 1977, 1986; Wood \& Bandura, 1989), has received increasing attention in the applied psychology literature over the past 2 decades. Meta-analytic investigations have shown that self-efficacy is positively related to performance in a variety of workrelated settings (Sadri \& Robertson, 1993; Stajkovic \& Luthans, 1998). In particular, self-efficacy has been shown to play a prominent role in training motivation, yielding positive associations with learning and performance throughout the training process and in posttraining transfer contexts (Colquitt, LePine, \& Noe, 2000).

Because self-efficacy is important to success in training, remediating low self-efficacy is a critical concern. Fortunately, a growing body of empirical literature has demonstrated that interventions involving behavior modeling can be designed to mitigate the negative effects of low self-efficacy (e.g., Gist, Schwoerer, \& Rosen, 1989; Gist, Stevens, \& Bavetta, 1991; Jones, 1986; Saks, 1995). Behavior modeling training stems from Bandura's $(1977,1986)$ social learning theory, which emphasizes applying observation, modeling, and 
vicarious reinforcement to changing human behavior (Goldstein \& Ford, 2002). In short, imitating the novel actions of others is a powerful way of learning, according to social learning theory (Goldstein \& Ford, 2002; see Taylor, Russ-Eft, \& Chan, 2005, for a meta-analytic review).

Behavior modeling is also viewed as one of the most viable means of remediating low selfefficacy (Haccoun \& Saks, 1998). For example, researchers have shown positive remediating effects of behavior modeling for persons low in pretraining self-efficacy with respect to the unemployed finding reemployment (Eden \& Aviram, 1993) and naval cadets overcoming seasickness (Eden \& Zuk, 1995). These studies essentially found attribute-treatment interactions between pretraining self-efficacy and behavior modeling such that behavior modeling had a positive effect on training outcomes for trainees low in pretraining self-efficacy, with little or no benefit for trainees high in pretraining self-efficacy.

The crux of the explanations for this interaction is that behavior modeling fosters confidence and provides adaptive strategies for skill development in persons who lack confidence and knowledge of how to develop their skills (i.e., those with low pretraining self-efficacy). However, behavior modeling is superfluous for persons already possessing confidence and adaptive skill development strategies (i.e., those with high pretraining selfefficacy).

Thus, the empirical literature has demonstrated that the normally handicapping effects of low selfefficacy can be remediated through interventions involving behavior modeling techniques. However, to our knowledge, no research has examined the extent to which low pretraining self-efficacy can be remediated in human-machine contexts, specifically with regard to complex skills that involve both cognitive and psychomotor demands.

The present laboratory study addresses this gap in the literature by examining the extent to which training in collaboration with a more experienced partner differentially affects trainees with low pretraining self-efficacy as compared with trainees with high pretraining self-efficacy. Because modeling and observation are crucial to collaborative learning, the fundamental premise of this study is that collaborative training protocols, in the same vein as behavior modeling training, are a viable means of remediating low pretraining self-efficacy.

\section{COLLABORATIVE LEARNING}

Terms such as collaborative learning, cooperative learning, group work, and peer learning have similar meanings (Bruffee, 1999). Collaborative learning generally describes an instructional technique in which individuals are placed in small groups or pairs while learning a specific task and are encouraged to communicate with their partners by sharing ideas and working together toward a common goal (Slavin, 1983).

Not only is collaborative learning a popular instructional technique, it also has support from a large body of empirical work (Johnson, Maruyama, Johnson, Nelson, \& Skon, 1981; Neo, 2003; Stanne, Johnson, \& Johnson, 1999; Wentzel, 2002). In addition to partners serving as models for one another, members of collaborative learning groups can collectively explore and elaborate on material and frequently develop better solutions to problems than any lone individual in the group (Ames \& Murray, 1982; Larson et al., 1985). After collaboration, individuals retain the problem-solving and skill development strategies they procured from their groups. Thus, working with a partner or partners facilitates individual mastery of the learning concepts.

Despite the widespread study of collaborative learning in the educational literature (Marr, 1997), very little research on collaboratively based training has been conducted in contexts concerning complex skills and human-machine interactions. However, research on active interlocked modeling by Shebilske, Regian, Arthur, and Jordan (1992) is one exception. Active interlocked modeling (AIM) is dyadic training described as observational learning in the context of actively performing a task in harmony with a partner (Arthur, Day, Bennett, McNelly, \& Jordan, 1997). The AIM protocol requires trainees to practice each half of a task alternately, with a partner practicing the other half.

Studies of AIM have shown that despite half the amount of hands-on practice with task controls and responsibilities, skill acquisition under AIM is equal to that under standard individual training (Shebilske et al., 1992). Such a result translates into a $100 \%$ gain in training efficiency over individual training. The increase in efficiency results from training two people simultaneously rather than one at a time, with no increase in trainer time or machine cost. Arthur et al. (1997) further demonstrated this efficiency effect with respect to 
skill retention and reacquisition after 8 weeks of nonpractice.

Research on the efficiency and effectiveness of AIM has employed the computer game Space Fortress as the criterion task. Space Fortress has a strong history as a research tool for studying complex skill acquisition (Donchin, 1989; Gopher, Weil, \& Bareket, 1994). It was designed to simulate the demands of dynamic aviation environments and other complex performance settings (Gopher, 1993). These demands include short- and longterm memory loading, high workload, decision making, prioritization, resource management, discrete motor responses, and difficult manual controls (Gopher, Weil, \& Siegel, 1989). In particular, success at Space Fortress requires dynamic attention control, as individuals must master the interplay between cognitive and psychomotor demands (Arthur et al., 1995; Shebilske, Goettl, \& Regian, 1999).

The present study also used Space Fortress and employed AIM as the collaborative training protocol. However, this study differs from previous studies in that novice AIM participants were paired with more experienced partners, whereas all previous studies paired novices with fellow novices. It is important to note that we did not pair collaborative trainees with more experienced partners who were considered experts. Rather, we paired collaborative trainees with more modestly skilled partners because of the concern that novices might not be able to communicate with and understand partners who are too advanced.

Research on scaffolding from the educational and developmental literatures directly speaks to issues of partner competence in collaborative learning settings. Scaffolding refers to instructional approaches that involve collaborating and providing just enough assistance to extend the learner's current capabilities. Assistance could come in the form of providing suggestions or asking thoughtprovoking questions, but typically scaffolding refers to modeling advanced behaviors that are within the reach of the learner's current capabilities.

Parallel participation (i.e., simply performing alongside one another) is not sufficient to enhance an individual's development. Rather, development occurs when individuals function in a coordinated manner through a joint understanding of the situation (Hogan \& Tudge, 1999; Rieber \& Carton, 1987; Vygotsky, 1978). If assistance is too far advanced or out of the reach of the individual's pre- sent capabilities, then joint understanding is not likely to occur.

\section{OVERVIEW OF STUDY AND HYPOTHESES}

Previous research has demonstrated the importance of pretraining self-efficacy as well as the effectiveness of learning protocols that involve behavior modeling as a means of mitigating the harmful effects of low self-efficacy on training outcomes. Therefore, examining the extent to which collaborative learning, which involves behavior modeling, can be used to address the negative influence of low self-efficacy is worthwhile. However, no such examinations to this date have been conducted. Furthermore, to our knowledge no research has examined the extent to which low pretraining self-efficacy can be remediated with respect to skills requiring human-machine interaction and both substantial cognitive and psychomotor demands.

Therefore, the purpose of this study was to examine the effectiveness of collaborative training for individuals with low pretraining self-efficacy versus individuals with high pretraining selfefficacy regarding the acquisition of a complex skill: a computer task that simulated the demands of a dynamic aviation environment. Participants underwent training in one of two conditions: individual training or collaborative training with a more experienced partner. Active interlocked modeling was employed as the collaborative training protocol.

Training consisted of two $2 \frac{1}{2}$-hr sessions separated by 1 week. The training manipulation occurred during the first session; thus, we were able to investigate both proximal and distal effects. Self-efficacy was measured prior to training, during training, and at the end of training. In general, it was expected that collaborative training would yield greater skill acquisition and mid- and posttraining self-efficacy than would individual training. However, we also expected an interaction between training condition and pretraining selfefficacy because of a differential need for boosts to self-efficacy and exposure to advanced performance strategies. Accordingly, we examined the following two specific hypotheses:

Hypothesis 1: The beneficial effects that collaborative training has on skill acquisition and mid- and posttraining self-efficacy relative to individual 
training will be stronger for individuals with low pretraining self-efficacy than for individuals with high pretraining self-efficacy.

Hypothesis 2: The differential influence of collaborative training on skill acquisition for individuals with low pretraining self-efficacy relative to individuals with high pretraining self-efficacy will be mediated by midtraining self-efficacy and practice performance.

\section{METHOD}

\section{Participants}

Two hundred twenty-three men enrolled in an introductory psychology course at the University of Oklahoma completed this study as part of a course research requirement. We chose to limit the study to male participants to control for the effects (i.e., social and interpersonal dynamics) associated with mixed-gender pairs in the collaborative condition. Participation was limited to righthanded volunteers due to hardware constraints.

Logistical constraints prevented us from using full random assignment to the training conditions; this study should be considered a quasiexperiment. Initially, participants were randomly assigned to the individual condition, to the collaborative condition, or to serve as more experienced partners for participants in the collaborative condition. However, 24 participants withdrew from the study. Consequently some participants who were originally assigned to the collaborative condition or to serve as more experienced partners had to be reassigned to the individual condition. This resulted in 87 participants training in the individual condition, 68 training in the collaborative condition, and 68 serving as the more experienced partners for the participants in the collaborative condition.

The more experienced partners underwent 3.5 hr of individual training ( $2.5 \mathrm{hr}$ on Day 1 of training and $1 \mathrm{hr}$ on Day 2) before they were paired with a novice from the collaborative condition. That is, at the time when collaboration began, the more experienced partners had already performed 32 more games of Space Fortress than their less experienced counterparts in the collaborative condition. At that time, the more experienced partners were performing on average more than two standard deviations $(d=2.31)$ higher than their novice partners.

As previously stated, the more experienced partners were by no means experts. Previous research with Space Fortress has shown that individuals typically perform an additional one to two standard deviations higher after $10 \mathrm{hr}$ of training versus $3.5 \mathrm{hr}$ (e.g., Arthur et al., 1997). Bonuses of $\$ 80, \$ 60, \$ 40, \$ 20$, and $\$ 10$ were awarded to the top 5 performers (more experienced partners received a commensurate bonus if their counterparts earned a bonus).

\section{Performance Task}

Laboratory rooms were equipped with a table, a computer and monitor, a right-hand joystick, a three-button mouse for the left hand, and two right-handed chair desks. In Space Fortress (Mané \& Donchin, 1989), trainees control a space ship's flight path using the joystick and shoot missiles with a trigger on the joystick. A fortress is located at the center of the screen and surrounded by two concentric hexagons.

An information panel at the bottom of the screen indicates fortress vulnerability, which changes with each missile hit. Friend and foe mines fly in the space surrounding the fortress and are identified by a mine indicator on the information panel. To destroy foe mines, trainees are required to push an "identify friend or foe" mouse button at the appropriate time. Symbols appear on the screen just below the fortress to indicate opportunities to gain bonus points or additional missiles by pushing either a "points" or "missiles" mouse button at the appropriate time.

The information panel also shows the number of available missiles, a battle score, and component scores based on ship velocity, ship control, and the speed of dispatching mines. The screen displays a total score, which is a composite of the others, along with more detailed performance feedback at the end of each game. More detailed descriptions and illustrations of Space Fortress can be found in Arthur et al. (1995) and Shebilske, Goettl, Corrington, and Day (1999).

\section{Self-Efficacy}

We used a task-specific self-efficacy scale based on sample items from scales used in several previous studies (e.g., Bell \& Kozlowski, 2002; Nease, Mudgett, \& Quiñones, 1999) as well as items developed specifically for this study. Although validity has been demonstrated for measures of generalized, trait-based self-efficacy (e.g., Chen, Gully, \& Eden, 2001), Bandura (1986, 1997) strongly argued for using task-specific measures, 
especially in the prediction of task performance. Our scale consisted of 12 items, including "I can meet the challenges of Space Fortress" and "I am confident that I have what it takes to perform Space Fortress well." Participants responded using a 5point scale $(1=$ strongly disagree, $5=$ strongly agree). We obtained coefficient alphas of .92, .92, and .95 for the three administrations of the scale, respectively.

\section{Procedure}

Participation took place on 2 days, 1 week apart, for approximately $2 \frac{1}{2} \mathrm{hr}$ each day. Participants first watched a 17-min training video detailing instructions and optimal strategies for performing Space Fortress. Afterward, they performed four 3-min baseline games of Space Fortress and then watched a 5-min video reviewing the instructions and optimal strategies. Baseline scores for individually trained participants $(M=-1,669.30, S D$ $=807.13$ ) were roughly equal to the scores for the participants in the collaborative training condition $(M=-1,566.63, S D=1,027.20), t(153)=-0.70$, $p=.49, d=-0.11$. Participants were then given the self-efficacy scale for the first time (i.e., pretraining). Self-efficacy scores for this first administration for participants in the individual condition $(M=2.90, S D=0.78)$ were roughly equal to the scores for the participants in the collaborative training condition $(M=2.89, S D=0.88), t(153)=0.04$, $p=.97, d=0.01$.

For the rest of the first day, participants completed two 10-game training sessions of Space Fortress (Sessions 1 and 2). The experimental manipulation occurred during these two training sessions. The first 8 games of every 10-game session were practice, and the last 2 games were test games. All games lasted 3 min. After Session 2, participants again completed the self-efficacy scale. One week later, participants returned for a second day of participation and completed a 2-game test of retention (Session 3 ) followed by three 10-game training sessions (Sessions 4, 5, and 6). After Session 6, participants completed the self-efficacy scale for the third and final time. Monetary bonuses were based solely on test game performance.

\section{Training Conditions}

Participants in the individual training condition performed all practice and test games individually, never working with a partner. Participants in the collaborative condition worked with a more experienced partner during the eight practice games of Sessions 1 and 2 (i.e., the first day of training), but they performed the test games of Sessions 1 and 2 individually without aid from their partners and trained individually for Sessions 3 through 6. During the practice games in Sessions 1 and 2, collaborative participants practiced with their partners by first controlling all functions related to the mouse while the more experienced partner controlled all functions related to the joystick, and they exchanged roles after every practice game. Communication during and between practice games was encouraged.

\section{RESULTS}

Table 1 shows the means, standard deviations, and correlations for self-efficacy and individual Space Fortress test scores. Self-efficacy scores were highly intercorrelated across the three administrations, mean $r=.69, p<.001$. Space Fortress scores

TABLE 1: Means, Standard Deviations, and Intercorrelations of Study Variables

\begin{tabular}{lrrrrrrrrrrr}
\hline Variable & $M$ & $S D$ & 1 & 2 & 3 & 4 & 5 & 6 & 7 & 8 & 9 \\
\hline 1. SE pretraining & 2.89 & 0.82 & - & & & & & & & & \\
2. SE midtraining & 3.50 & 0.77 & .71 & - & & & & & & \\
3. SE posttraining & 3.68 & 0.94 & .56 & .79 & - & & & & & & \\
4. SF baseline & $-1,624.30$ & 908.55 & .50 & .43 & .42 & - & & & & & \\
5. SF Session 1 & 319.17 & $1,472.77$ & .40 & .46 & .44 & .73 & - & & & & \\
6. SF Session 2 & 734.97 & $1,602.91$ & .39 & .53 & .52 & .66 & .87 & - & & & \\
7. SF Session 3 & 380.97 & $1,374.57$ & .33 & .43 & .43 & .65 & .84 & .88 & - & & \\
8. SF Session 4 & $1,400.05$ & $1,766.99$ & .38 & .52 & .55 & .61 & .81 & .89 & .86 & - & \\
9. SF Session 5 & $1,752.07$ & $1,985.91$ & .42 & .52 & .59 & .61 & .79 & .86 & .83 & .91 & -.93 \\
10. SF Session 6 & $1,943.11$ & $1,955.04$ & .38 & .52 & .59 & .60 & .80 & .87 & .83 & .93 & .93 \\
\hline
\end{tabular}

Note. SE = self-efficacy. SF = Space Fortress. Session $3=$ Test of retention after a 1-week nonpractice interval. All values are significant at .001 . All tests are two-tailed. $N=155$. 
were highly intercorrelated across sessions, mean $r=.80, p<.001$. As expected, the correlations between self-efficacy and Space Fortress scores were positive and statistically significant, mean $r=.46$, $p<.001$.

\section{Skill Acquisition}

To test Hypothesis 1 and examine the extent to which collaborative training differentially affected skill acquisition in individuals with low pretraining self-efficacy versus those with high pretraining self-efficacy, we conducted a 2 (pretraining selfefficacy: low vs. high) $\times 2$ (training condition: individual vs. collaborative) $\times 7$ (training session) mixed ANOVA. Low and high self-efficacy were operationalized using the bottom and top thirds ( $M \mathrm{~s}=1.91$ and 3.83, respectively) of the pretraining self-efficacy score distribution. We also conducted a similar mixed ANOVA $(3 \times 2 \times 7)$ that included the middle third $(M=2.91)$ of the pretraining self-efficacy score distribution. Overall, the main effects and interactions with and without the middle third were similar, and the effects of training condition on performance scores for the middle third of the distribution mirrored those for the top third.

Means, standard deviations, and standardized differences are shown in Table 2 . The results showed main effects for training session, $F(6$, $546)=295.98, p<.001, \eta^{2}=.77$, and self-efficacy, $F(1,91)=31.34, p<.001, \eta^{2}=.26$. The main effect for training condition did not reach statistical significance, $F(1,91)=3.35, p=.07, \eta^{2}=.04$. However, the results revealed a statistically significant two-way interaction between session and training condition, $F(6,546)=2.68, p<.05, \eta^{2}=.03$. Participants in the collaborative condition experienced greater gains in skill acquisition than did participants in the individual condition. The results also revealed a statistically significant two-way interaction between session and self-efficacy; $F(6$, $546)=5.80, p<.001, \eta^{2}=.06$. Participants with high pretraining self-efficacy experienced greater gains in skill acquisition from baseline to the end of training than did those with low pretraining selfefficacy.

Although the results did not reveal a statistically significant two-way interaction between selfefficacy and condition, $F(1,91)=0.18, p=.68, \eta^{2}<$ .01 , there was a statistically significant three-way interaction among session, self-efficacy, and condition, $F(6,546)=3.30, p<.01, \eta^{2}=.04$, show- ing that collaborative training yielded different effects for participants with low pretraining selfefficacy than for participants with high pretraining self-efficacy. As shown in Table 2, the differences in scores on the second day of training between collaboratively and individually trained participants with low self-efficacy were consistently stronger than the differences in scores on the first day of training. This was not the case for participants with high self-efficacy: There was no growing trend in score differences between collaboratively and individually trained participants with high selfefficacy across the 2 days of training.

These results are consistent with Hypothesis 1 and show that beneficial effects of collaborative training occurred for participants with low pretraining self-efficacy but not for those with high pretraining self-efficacy. However, the extent of this differential effect of collaborative training as a function of pretraining self-efficacy is somewhat confounded by differences in baseline scores. As shown in Table 2, high self-efficacy participants in the collaborative condition had higher baseline scores than high self-efficacy participants in the individual condition $(d=0.48)$, whereas low selfefficacy participants in the collaborative condition had lower baseline scores than low self-efficacy participants in the individual condition $(d=-0.27)$. Because of this nonequivalence in baseline scores, these results could be viewed as an underestimate of the differential effect that collaborative training had on participants with low pretraining selfefficacy as compared with those with high pretraining self-efficacy.

To address this nonequivalence, we conducted two separate 2 (training condition: individual vs. collaborative) $\times 6$ (training session) ANCOVAs with baseline scores as the covariate. The first analysis was conducted for participants low on pretraining self-efficacy. The second analysis was conducted for participants high on pretraining selfefficacy. Conducting separate ANCOVAs for low and high pretraining self-efficacy adjusts for the difference in nonequivalence of baseline scores between collaborative and individual conditions as a function of pretraining self-efficacy without removing the overall effects of pretraining selfefficacy, which would occur with a 2 (condition) $\times$ 2 (self-efficacy) $\times 6$ (session) ANCOVA. The adjusted means are shown in Figure 1, and the adjusted standardized differences are shown in Table 2. 


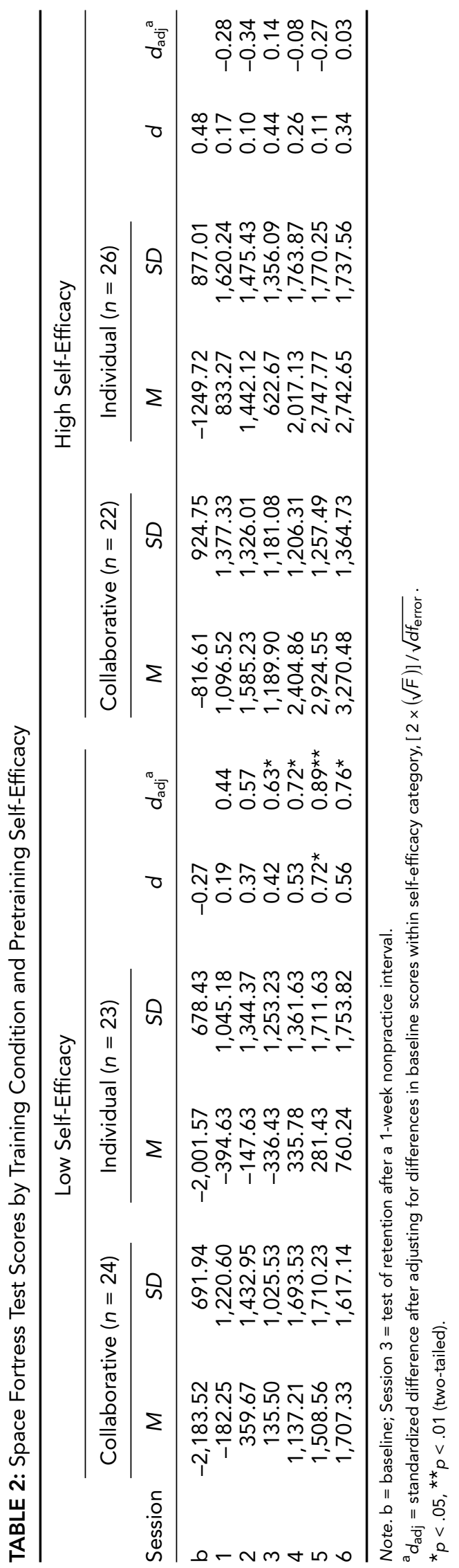




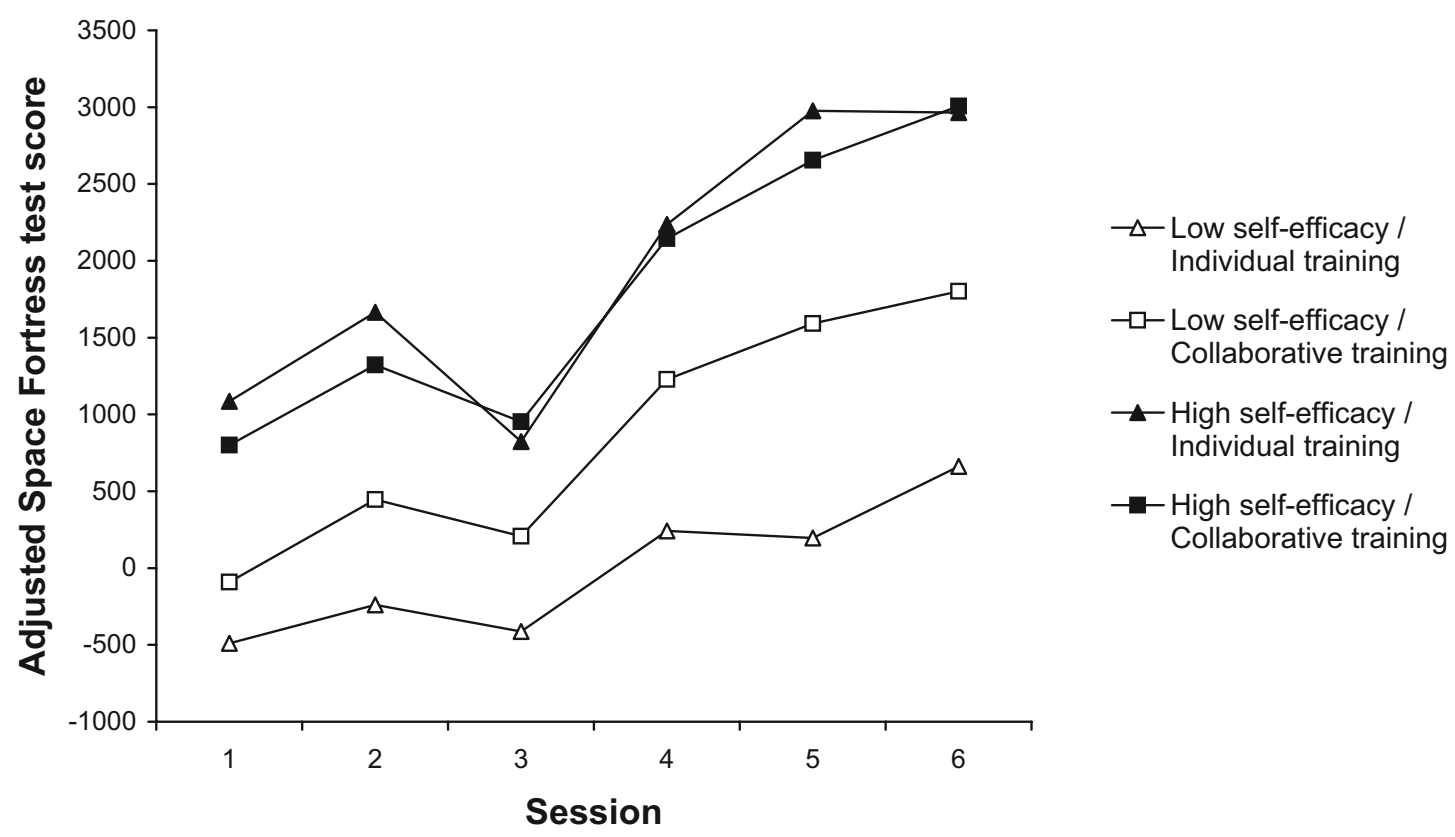

Figure 1. Mean skill acquisition scores across sessions as a function of self-efficacy and training condition, adjusted for differences in baseline scores within self-efficacy category.

The results of these analyses revealed a stronger differential effect for collaborative training as compared with the previous analyses. In support of Hypothesis 1, the results for participants with the low pretraining self-efficacy revealed a statistically significant main effect for training condition, $F(1,44)=6.77, p<.05, \eta^{2}=.13$, whereas the results for participants with high pretraining selfefficacy did not reveal a statistically significant main effect for training condition, $F(1,45)=0.26$, $p=.62, \eta^{2}<.01$. As shown in Table 2 , the adjusted standardized differences for participants with the low pretraining self-efficacy were moderate to strong and consistently in favor of collaborative training; differences for participants with high pretraining self-efficacy were weak and not consistently in favor of either training condition.

\section{Mid- and Posttraining Self-Efficacy}

To examine the interaction proposed in Hypothesis 1 with respect to mid- and posttraining selfefficacy scores, we conducted similar analyses to those discussed previously for skill acquisition scores. Specifically, we conducted a 2 (pretraining self-efficacy: low vs. high) $\times 2$ (training condition: individual versus collaborative $) \times 3$ (administration) ANOVA as well as separate 2 (training condition: individual vs. collaborative) $\times 3$ (admin- istration) ANCOVAs with baseline Space Fortress scores as the covariate for participants low and high on pretraining self-efficacy. Means, standard deviations, standardized differences, and adjusted standardized differences are shown in Table 3. Figure 2 shows the adjusted means.

The results of the ANOVA revealed statistically significant main effects for administration, $F(2$, $182)=65.40, p<.001, \eta^{2}=.42$, and self-efficacy, $F(1,91)=263.41, p<.001, \eta^{2}=.74$, as well as a significant two-way interaction between administration and self-efficacy, $F(2,182)=11.50, p<$ $.001, \eta^{2}=.11$. The pattern of means showed that participants with low pretraining self-efficacy slightly benefited from collaborative training with respect to mid- and posttraining self-efficacy scores, whereas participants with high pretraining self-efficacy did not benefit from collaborative training. However, neither the two-way interaction between training condition and pretraining selfefficacy, $F(1,91)=0.32, p=.57, \eta^{2}<.01$, nor the three-way interaction among administration, condition, and self-efficacy, $F(2,182)=0.71, p=.49$, $\eta^{2}<.01$, was statistically significant. Analyses involving the adjusted means yielded similar results. Overall, the results involving mid- and posttraining self-efficacy scores did not support Hypothesis 1. 


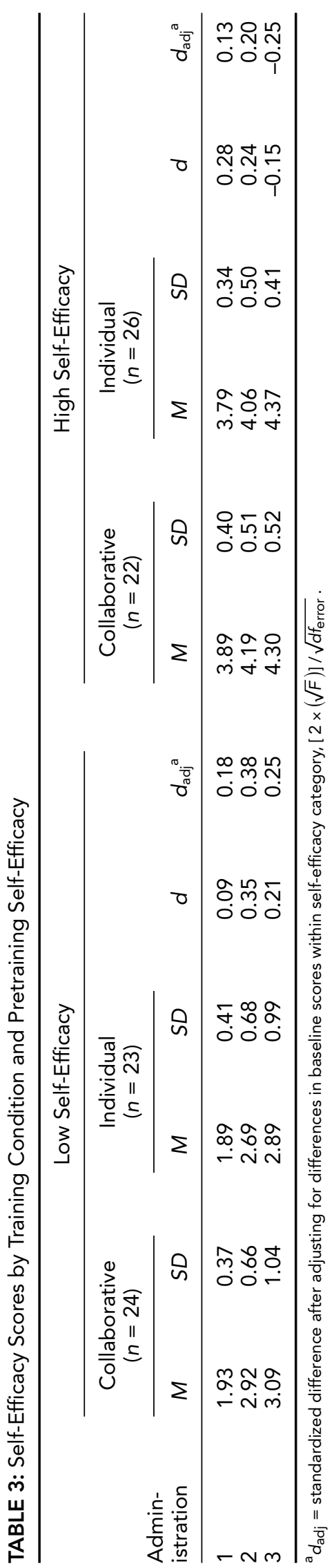




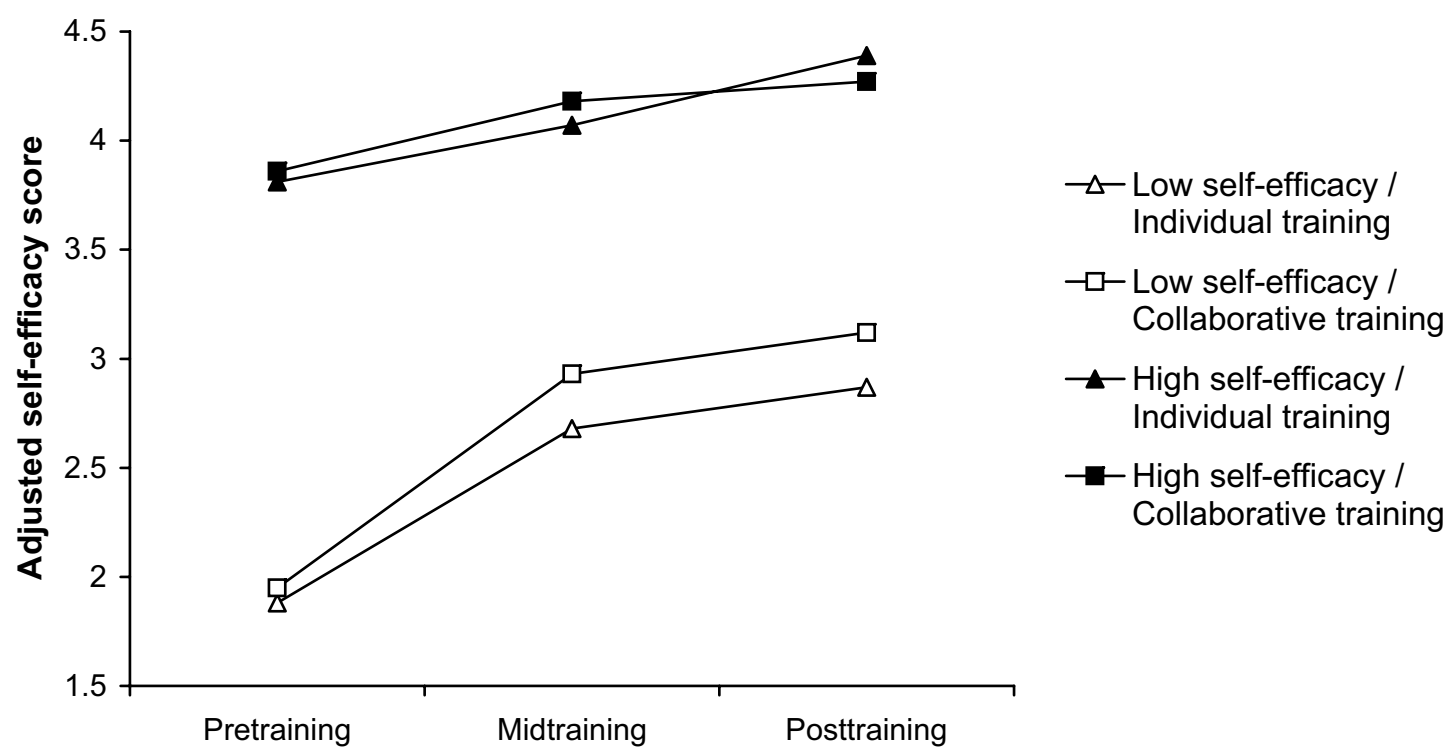

Administration

Figure 2. Mean self-efficacy scores across administrations as a function of self-efficacy and training condition, adjusted for differences in baseline scores within self-efficacy category.

\section{Practice}

We predicted in Hypothesis 2 that practice performance would serve as a mediator of the differential influence of collaborative training on skill acquisition for individuals with low pretraining self-efficacy. As such, before testing for mediation, we first examined the interaction between training condition and pretraining self-efficacy with respect to practice scores. Using the average of the eight practice game scores from Sessions 1 and 2 (when the training manipulation took place), we conducted analyses similar to those for skill acquisition and self-efficacy scores. Specifically, we conducted a 2 (pretraining self-efficacy: low vs. high) $\times 2$ (training condition: individual vs. collaborative) $\times 3$ (training session including baseline scores) ANOVA as well as separate 2 (training condition: individual versus collaborative) $\times 2$ (training session) ANCOVAs with baseline Space Fortress scores as the covariate for participants low and high on pretraining self-efficacy.

Means, standard deviations, standardized differences, and adjusted standardized differences are shown in Table 4. The adjusted means are shown in Figure 3. Regarding the ANOVA, the results showed effects similar to those found for individual skill acquisition scores. In particular, there was a statistically significant three-way interaction among session, self-efficacy, and training condition, $F(2,182)=6.18, p<.01, \eta^{2}=.06$, showing that collaborative training yielded different practice effects for participants with low pretraining self-efficacy than for participants with high pretraining self-efficacy. Specifically, participants with low pretraining self-efficacy who trained collaboratively experienced substantially higher performance levels during practice than did participants with low pretraining self-efficacy who trained individually (average $d=1.60$ ).

Although similar benefits took place for participants with high pretraining self-efficacy who trained collaboratively, the results did not lead to as large a difference in performance levels experienced during practice (average $d=0.92$ ). As before, because of the differential nonequivalence in baseline scores, these results could be viewed as an underestimate of the differential effect that collaborative training had on participants with low pretraining self-efficacy as compared with participants with high pretraining self-efficacy.

The results of the ANCOVAs controlling for baseline scores within self-efficacy category showed that the performance benefits experienced during collaborative practice for participants with low pretraining self-efficacy, $F(1,44)=37.98, p<$ $.001, \eta^{2}=.46$, were substantially larger than those experienced by participants with high pretraining 


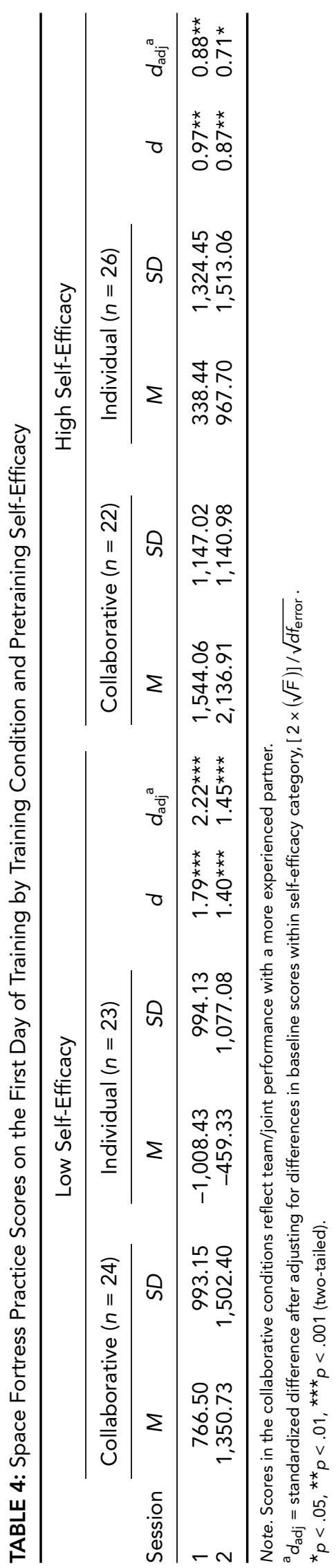

Downloaded from hfs.sagepub.com at UNIV OF OKLAHOMA on January 20, 2016 


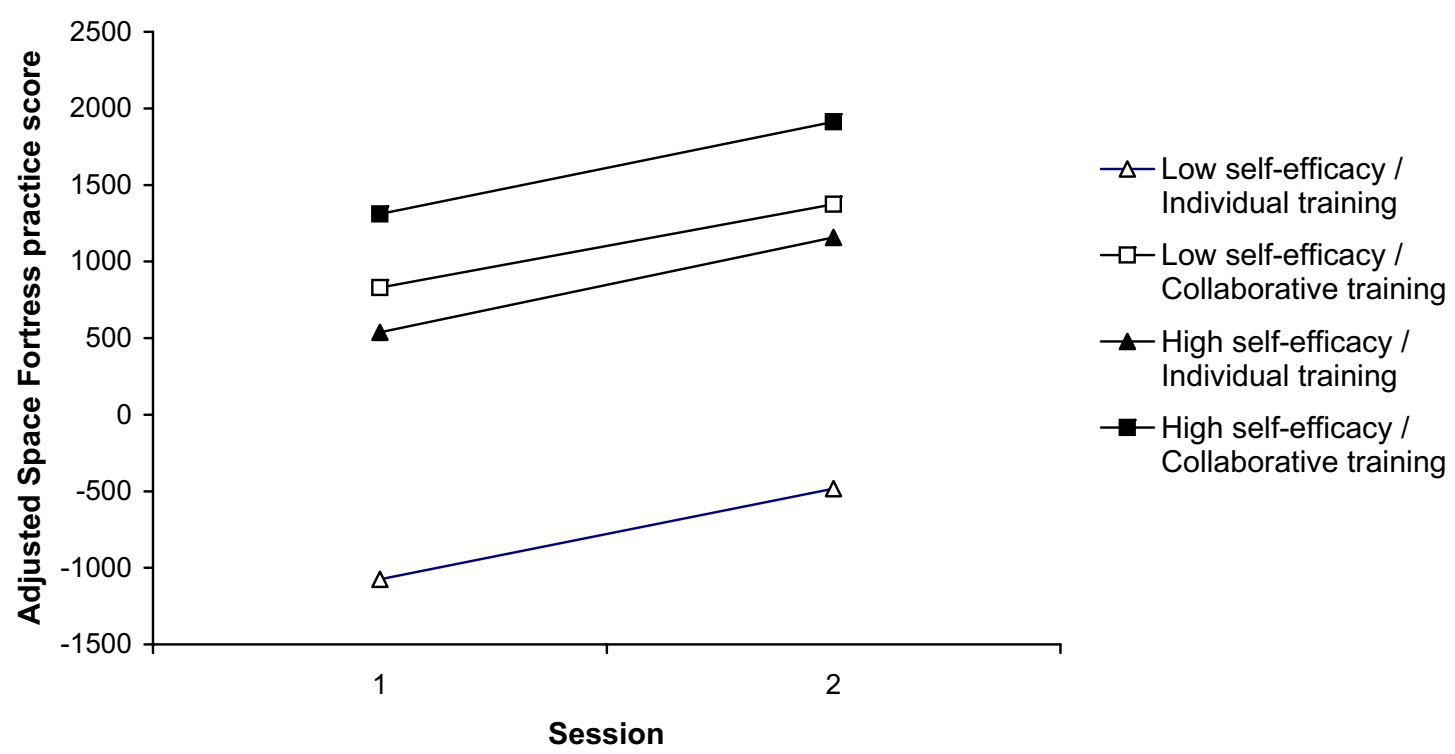

Figure 3. Mean practice scores across sessions as a function of self-efficacy and training condition, adjusted for differences in baseline scores within self-efficacy category. Scores in the collaborative conditions reflect team/joint performance with a more experienced partner.

self-efficacy, $F(1,45)=7.70, p<.01, \eta^{2}=.15$. Like the results for individual skill acquisition scores, these results further support Hypothesis 1 and demonstrate the differential effects that collaborative training with a more experienced partner can have, depending on trainees' pretraining self-efficacy.

\section{Mediation}

We used regression analyses to test Hypothesis 2 and investigate the extent to which practice scores mediated the differential effect that collaborative training had on test scores for individuals with low pretraining self-efficacy relative to individuals with high pretraining self-efficacy. Because there was no significant interaction for mid- and posttraining self-efficacy scores, the conditions for examining the mediating role of self-efficacy were not met.

Using the entire sample, we first replicated the interaction effect that pretraining self-efficacy and training condition had on practice scores, as previously reported using ANOVA and ANCOVA procedures. As shown in Table 5, the Pretraining Self-Efficacy $\times$ Training Condition interaction made a statistically significant contribution $(\beta=$ $-.22, p<.01)$ to practice scores on the first day of training after controlling for the effects of base- line scores, pretraining self-efficacy, and training condition.

Next, we tested the contribution that practice scores had on individual test scores on the first day of training after controlling for the effects of baseline scores, pretraining self-efficacy, training condition, and the Pretraining Self-Efficacy $x$ Training Condition interaction. As shown in Table 6 , practice scores made a statistically significant contribution $(\beta=.69, p<.01)$ to individual test scores. Regarding mediation, the Pretraining Self-Efficacy $\times$ Training Condition interaction was significantly related to individual test scores before practice scores were included in the regression model $(\beta=-.21, p<.05)$. However, this interaction was no longer statistically significant after practice scores were included $(\beta=-.06, p=$ .41). In support of Hypothesis 2, these findings indicate that practice scores mediated the differential influence on individual skill acquisition that collaborative training had on individuals with low pretraining self-efficacy relative to individuals with high pretraining self-efficacy.

\section{DISCUSSION}

The present study extends the literature on selfefficacy and training by demonstrating how a collaborative training protocol (i.e., AIM) mitigated 
TABLE 5: Regression Results for Space Fortress Practice Scores on the First Day of Training

\begin{tabular}{lllll}
\hline Model & $\beta_{1}$ & $\beta_{2}$ & $R^{2}$ & $\Delta R^{2}$ \\
\hline 1. SF baseline & $.54^{\star \star \star}$ & $.57^{\star \star \star}$ & $.53^{\star \star \star}$ & \\
Pretraining self-efficacy (A) & .08 & $.22^{\star \star}$ & & \\
Training condition (B) & $.40^{\star \star \star}$ & $.40^{\star \star \star}$ & & \\
2. A $\times$ B & & $-.22^{\star \star}$ & $.55^{\star \star \star}$ & $.02^{\star \star}$ \\
\hline
\end{tabular}

Note. $\beta_{1}=$ standardized regression coefficient in the first model. $\beta_{2}=$ standardized regression coefficient in the second, final model. $R^{2}=$ proportion of variance accounted for in the dependent variable by the set of predictors in the regression equation. $\Delta R^{2}=$ incremental variance accounted for by the additional step in the regression equation. SF $=$ Space Fortress. Training condition: individual $=0$, collaborative $=1 . \mathrm{N}=155$.

${ }^{\star *} p<.01 ;{ }^{* \star *} p<.001$.

the normally deleterious effects of low pretraining self-efficacy on skill acquisition for a complex task that involved strong cognitive and psychomotor demands. Previous studies on remediating low pretraining self-efficacy have not involved such tasks and also have not examined the viability of collaborative training as a remediating intervention. Rather, previous research has focused more exclusively on the effectiveness of modeling techniques on predominantly cognitive tasks (e.g., computer software skills, Gist et al., 1989), or the primary dependent variable in previous studies involved adjustment (e.g., overcoming seasickness, Eden \& Zuk, 1995; beginning a new job, Saks, 1995; and finding reemployment, Eden \& Aviram, 1993) rather than actual skill acquisition.

Importantly, the beneficial effects of collaborative training for low self-efficacious participants observed in this study were more pronounced on tests of further skill acquisition taken 1 week after collaborative training as compared with tests taken immediately after collaborative training. This finding is consistent with Taylor et al.'s (2005) metaanalytic findings for behavior modeling training, which showed that delayed posttests reflected greater benefits of behavior modeling training on skills and job performance as compared with posttests taken immediately after training. Similar to individuals who undergo behavior modeling training, individuals who train collaboratively may continue to improve their skills over time as they continue to utilize the learning strategies they acquired in training. Together, our findings and those of Taylor et al. (2005) reinforce the argument that immediate tests of knowledge and skill often do not adequately reflect learning and are insufficient in examinations of training effectiveness (Schmidt \& Björk, 1992).

The interaction observed between pretraining self-efficacy and training condition regarding skill acquisition supports previous research on behavioral plasticity effects and interventions designed

TABLE 6: Regression Results for Space Fortress Test Scores on the First Day of Training

\begin{tabular}{lllll}
\hline Model & $\beta_{1}$ & $\beta_{2}$ & $R^{2}$ & $\Delta R^{2}$ \\
\hline 1. SF baseline & $.59^{\star \star \star}$ & $.20^{\star \star}$ & & \\
Pretraining self-efficacy (A) & $.25^{\star \star}$ & .10 & & \\
Training condition (B) & .05 & $-.22^{\star \star}$ & & \\
A $\times$ B & $-.21^{\star}$ & -.06 & $.42^{\star \star \star}$ & \\
$\begin{array}{l}\text { 2. SF practice on the first day } \\
\text { of training }\end{array}$ & & $.69^{\star \star \star}$ & $.64^{\star \star \star}$ & $.22^{\star \star \star}$ \\
\hline
\end{tabular}

Note. $\beta_{1}=$ standardized regression coefficient in the first model. $\beta_{2}=$ standardized regression coefficient in the second, final model. $R^{2}=$ proportion of variance accounted for in the dependent variable by the set of predictors in the regression equation. $\Delta R^{2}=$ incremental variance accounted for by the additional step in the regression equation. SF $=$ Space Fortress. Training condition: individual $=0$, collaborative $=1 . N=155$.

${ }^{\star} \mathrm{p}<.05,{ }^{\star \star} \mathrm{p}<.01,{ }^{\star \star \star} \mathrm{p}<.01$. 
to mitigate the typically harmful influence of low self-efficacy. Behavioral plasticity is the notion that certain individuals are lacking in some quality critical to adaptive behavior and are thus more susceptible than others to external/social influences. Plasticity effects were initially demonstrated with self-esteem and workplace behaviors - with persons low on self-esteem being more "behaviorally plastic" than persons high on self-esteem (Brockner, 1988) - and the results of the present study corroborate previously observed plasticity effects for self-efficacy. However, future research is needed to better isolate the causal forces behind interventions designed to remediate low selfefficacy.

Specifically, collaborative learning protocols such as AIM involve two processes implicated as critical to the success of behavior modeling training, especially with respect to mitigating the harmful effects of low pretraining self-efficacy. These processes are vicarious experiences and mastery experiences (Eden \& Aviram, 1993; Haccoun \& Saks, 1998). Vicarious experiences involve observing the actions and outcomes of another person performing a task. Indeed, observational learning accounts for much of the effects associated with AIM (Day, Arthur, Paulus, \& Fein, 2003; Shebilske, Jordan, Goettl, \& Paulus, 1998).

Mastery experiences, sometimes called enactive attainment, refer to practice opportunities that allow individuals to focus attention on activities that directly support higher levels of task performance that have yet to be experienced. Mastery experiences not only provide trainees with a taste for success, they also give trainees opportunities to participate in activities that enable skill development. Mastery experiences are apparent in the AIM protocol in that trainees (a) are able to better focus their attention and cognitive resources on task requirements as they perform half of a task while their partners perform the other half and (b) experience higher levels of practice performance as compared with individual trainees because task responsibilities are more easily performed as they are split between partners (Day, Arthur, \& Shebilske, 1997).

In general, with collaborative learning individuals are able to share and explore ideas with their partners and consequently develop performance strategies that might not develop, or might not develop as quickly, if individuals were learning by themselves. In other words, with the help of col- laborative partners, individuals are able to function at more advanced levels than they are capable of independently, especially when help comes from a more experienced partner (Tudge, 1992). Thus, mastery experiences allow an individual to practice and internalize advanced aspects of a skill, which in turn elevates the individual's performance level (Tudge, 1992).

In the present study, we were unable to isolate either mastery or vicarious experiences. Thus, questions remain to be tested. For instance, what are the relative contributions of vicarious and mastery experiences to plasticity effects? Is one more important than the other, or are they both necessary but insufficient conditions?

Likewise, future research is needed to better identify the variables that mediate such interventions. In this study, no differential effects were observed for mid- and posttraining self-efficacy, but a differential effect was observed for practice performance. This suggests that collaborative learning enhanced skill acquisition without first raising self-efficacy and that collaborative learning may first enhance task knowledge and skill development strategies that directly increase skill acquisition.

Perhaps such gains in skill acquisition would subsequently build later confidence. Indeed, some researchers (e.g., Gist et al., 1989) have indicated that enhanced self-efficacy from behavior modeling may come as a result of enhanced performance. Such may be the case in this study, with the differential effects of collaborative training on later self-efficacy occurring more slowly than for actual skill acquisition. Our observed pattern of means is consistent with this explanation. If later observations had been made, perhaps significant effects in subsequent self-efficacy would have been found.

More generally, the present findings suggest that the causal roles played by self-efficacy may not be as straightforward and direct as many might think. In fact, there is currently a debate in the literature regarding causality between self-efficacy and performance (Heggestad \& Kanfer, 2005), and recent meta-analytic research has even demonstrated that self-efficacy offers little if any main effect on performance after controlling for prior performance and other individual differences related to motivation and performance (Judge, Jackson, Shaw, Scott, \& Rich, 2007). However, the causal roles played by self-efficacy may be 
more apparent in terms of how it interacts with other variables of interest (Judge et al., 2007), which is what we found in the present study. The debate concerning causality is presently limited to investigations involving main effects, and more research on the moderating role of self-efficacy is needed.

A primary implication of this study is that collaborative learning can be effective for skill training, particularly for trainees with low pretraining self-efficacy. The differential effects of collaborative learning as a function of self-efficacy highlight the importance of person analysis and tailoring training to meet differing trainee needs. Individuals with low self-efficacy often struggle with new and complex tasks, and the acquisition of such skills could be facilitated for trainees with low self-efficacy by providing opportunities to collaborate with more experienced partners.

Depending on logistical issues, such an intervention may be a more feasible and less costly alternative to traditional behavior modeling techniques, particularly when training involves a small number of trainees. However, the feasibility of pairing novice trainees with more experienced partners decreases as the number of trainees increases (Davis \& Yi, 2004). An alternative approach would be to team novice trainees together in a collaborative structure, but the extent to which such collaboration remediates low selfefficacy is unknown and warrants future research.

The more general question is whether the effectiveness of pairing novices in collaborative training is moderated by the level of expertise of their partners. Although it is often assumed that help and advice should come from experts, empirical research has demonstrated that a target task may be more easily learned when help comes from beginners because novices may have difficulty understanding the advanced and frequently abstract statements received from experts (Hinds, Patterson, \& Pfeffer, 2001).

As previously mentioned, we chose to pair novices with more modestly skilled partners to sufficiently stretch the understanding and capabilities of the novices without presenting demands that were too far advanced (Rieber \& Carton, 1987; Vygotsky, 1978). Consequently, it is not known whether the remediating effects of collaboration for individuals with low pretraining self-efficacy would have been different if collaborative trainees had been paired with fellow novices rather than more skilled partners. We speculate that similar effects might occur but that the effects would be weaker, given that there would be less scaffolding with novice partners. Nevertheless, future research on self-efficacy and collaborative training that involves manipulations of partner expertise is warranted.

Another avenue for future research is the extent to which gains for novices with low self-efficacy come at the expense of their partners. Although we were unable to fully examine the effects of collaboration on the more experienced partners, ancillary analyses on the individual performance of the more experienced partners after collaboration showed no significant correlation between their performance scores and the pretraining selfefficacy scores of their less experienced counterparts $(r=.07, p=.56)$.

It should also be noted that although the present results revealed a positive gain from collaborative learning for persons with low pretraining selfefficacy, the gains did not bring the skill acquisition levels of these persons to the levels found for persons with high pretraining self-efficacy. Thus, collaborative learning only lessened, rather than fully removed, the negative effects of low selfefficacy. Future research examining the extent to which the negative effects of low pretraining selfefficacy on complex skill acquisition can be more fully remediated is needed. In a similar vein, future research on the relative effectiveness of collaborative learning versus traditional behavior modeling and some combination of the two approaches would be worthwhile.

Finally, because training involved a laboratory task with a sample of male college students, generalization of this study's findings to real-world training environments should be made with caution. Future research on collaborative learning in real-world training contexts, including military and civilian work settings with broader samples and female participants, is warranted.

\section{Conclusion}

Individuals who enter training with low selfefficacy often struggle with learning new and complex tasks. Fortunately, there is a growing body of empirical literature demonstrating the usefulness of behavior modeling in mitigating the negative effects of low self-efficacy. The present study contributes to this literature by showing that collaborative training can similarly be an effective 
strategy for facilitating the skill acquisition process for trainees with low self-efficacy. Specifically, our results showed that collaborative training with a more experienced partner enhanced skill acquisition for individuals with low pretraining self-efficacy but not so much for individuals with high pretraining self-efficacy. We hope this study stimulates future research on remediation strategies for individuals with low self-efficacy and the extent to which other individual differences moderate the effectiveness of training programs utilizing modeling strategies.

\section{ACKNOWLEDGMENTS}

Part of this research was conducted for Rachel Sherwin's senior honors thesis at the University of Oklahoma under the supervision of Eric Day.

\section{REFERENCES}

Ames, G. J., \& Murray, F. B. (1982). When two wrongs make a right: Promoting cognitive change by social conflict. Developmental Psychology, 18, 894-897.

Arthur, W., Jr., Day, E. A., Bennett, W., Jr., McNelly, T. L., \& Jordan, J. A. (1997). Dyadic versus individual training protocols: Loss and reacquisition of a complex skill. Journal of Applied Psychology, 82, 783-791.

Arthur, W., Jr., Strong, M. H., Jordan, J. A., Williamson, J. E., Shebilske, W. L., \& Regian, J. W. (1995). Visual attention: Individual differences in training and predicting complex task performance. Acta Psychologica, 88, 3-23.

Bandura, A. (1977). Social learning theory. Englewood Cliffs, NJ: Prentice-Hall.

Bandura, A. (1986). Social foundations of thought and action: A social cognitive theory. Englewood Cliffs, NJ: Prentice-Hall.

Bandura, A. (1997). Self-efficacy: The exercise of control. New York: Freeman.

Bell, B. S., \& Kozlowski, W. J. (2002). Goal orientation and ability: Interactive effects on self-efficacy, performance and knowledge. Journal of Applied Psychology, 87, 497-505.

Brockner, J. (1988). Self-esteem at work: Research, theory, and practice. Lexington, MA: Lexington Books.

Bruffee, K. A. (1999). Collaborative learning. Baltimore: John Hopkins University Press.

Chen, G., Gully, S. M., \& Eden, D. (2001). Validation of a new general self-efficacy scale. Organizational Research Methods, 4, 62-83.

Colquitt, J. A., LePine, J. A., \& Noe, R. A. (2000). Toward an integrative theory of training motivation: A meta-analytic path analysis of 20 years of research. Journal of Applied Psychology, 85, 678-707.

Davis, F. D., \& Yi, M. Y. (2004). Improving computer skill training: Behavior modeling, symbolic mental rehearsal, and the role of knowledge structures. Journal of Applied Psychology, 89, 509-523.

Day, E. A., Arthur, W., Jr., Paulus, L. E., \& Fein, E. C. (2003). Dyadic protocols, observational learning, and the acquisition of complex skills. In Proceedings of the Human Factors and Ergonomics Society 47th Annual Meeting (pp. 2050-2053). Santa Monica, CA: Human Factors and Ergonomics Society.

Day, E. A., Arthur, W., Jr., \& Shebilske, W. L. (1997). Ability determinants of complex skill acquisition: Effects of training protocol. Acta Psychologica, 97, 145-165.

Donchin, E. (1989). The learning strategies project: Introductory remarks. Acta Psychologica, 71, 1-15.

Eden, D., \& Aviram, A. (1993). Self-efficacy training to speed reemployment: Helping people to help themselves. Journal of Applied Psychology, 78, 352-360.
Eden, D., \& Zuk, Y. (1995). Seasickness as a self-fulfilling prophecy: Raising self-efficacy to boost performance at sea. Journal of Applied Psychology, 80, 628-635.

Gist, M. E., Schwoerer, C., \& Rosen, B. (1989). Effects of alternative training methods on self-efficacy and performance in computer software training. Journal of Applied Psychology, 74, 884-891.

Gist, M. E., Stevens, C. K., \& Bavetta, A. G. (1991). Effects of selfefficacy and post-training intervention on the acquisition and maintenance of complex interpersonal skills. Personnel Psychology, 44, $837-861$.

Goldstein, I. L., \& Ford, J. K. (2002). Training in organizations: Needs assessment, development, and evaluation (4th ed.). Belmont, CA: Wadsworth.

Gopher, D. (1993). The skill of attention control: Acquisition and execution of attention strategies. In D. Meyer \& S. Korenblom (Eds.), Attention and performance XIV: Synergies in experimental psychology, artificial intelligence, and cognitive neuroscience (pp. 299-322). Cambridge, MA: MIT Press.

Gopher, D., Weil, M., \& Bareket, T. (1994). The transfer of skill from a computer game trainer to actual flight. Human Factors, 36, 387-405.

Gopher, D., Weil, M., \& Siegel, D. (1989). Practice under changing priorities: An approach to the training of complex skills. Acta Psychologica, 71, 147-177.

Haccoun, R. R., \& Saks, A. M. (1998). Training in the 21st century: Some lessons from the last one. Canadian Psychology, 39, 33-51.

Heggestad, E. D., \& Kanfer, R. (2005). The predictive validity of selfefficacy in training performance: Little more than past performance. Journal of Experimental Psychology: Applied, 11, 84-97.

Hinds, P. J., Patterson, M., \& Pfeffer, J. (2001). Bothered by abstraction: The effect of expertise on knowledge transfer and subsequent novice performance. Journal of Applied Psychology, 86, 1232-1243.

Hogan, D. M., \& Tudge, J. R. H. (1999). Implications of Vygotsky's theory for peer learning. In A. M. O'Donnell \& A. King (Eds.), Cognitive perspectives on peer learning (pp. 39-65). Mahwah, NJ: Erlbaum.

Johnson, D. W., Maruyama G., Johnson, R., Nelson, D., \& Skon, L. (1981). Effects of cooperative, competitive, and individualistic goal structures on achievement: A meta-analysis. Psychological Bulletin, $89,47-62$.

Jones, G. R. (1986). Socialization tactics, self-efficacy, and newcomers adjustments to organizations. Academy of Management Journal, 29, 262-279.

Judge, T. A., Jackson, C. L., Shaw, J. C., Scott, B. A., \& Rich, B. L. (2007). Self-efficacy and work-related performance: The integral role of individual differences. Journal of Applied Psychology, 92, $107-127$.

Larson, C., Dansereau, D., O'Donnell, A., Hythecker, V., Lambiotte, J., \& Rocklin, T. (1985). Effects of metacognitive and elaborative activity on cooperative learning and transfer. Contemporary Educational Psychology, 10, 342-348.

Mané, A. M., \& Donchin, E. (1989). The Learning Strategies program: An examination of the strategies in skill acquisition. Acta Psychologica, 71, 17-22.

Marr, M. B. (1997). Cooperative learning: A brief review. Reading \& Writing Quarterly: Overcoming Learning Difficulties, 13, 7-20.

Nease, A. A., Mudgett, B. A., \& Quiñones, M. A. (1999). Relationships among feedback sign, self-efficacy, and acceptance of performance feedback. Journal of Applied Psychology, 84, 806-814.

Neo, M. (2003). Developing a collaborative learning environment using a Web-based design. Journal of Computer Assisted Learning, $19,462-473$.

Rieber, R. W., \& Carton, A. S. (Eds.). (1987). The collected works of L. S. Vygotsky: Vol. 1. Problems of general psychology (N. Minick, Trans.). New York: Plenum Press.

Sadri, G., \& Robertson, I. T. (1993). Self-efficacy and work-related behavior: A review and meta-analysis. Applied Psychology: An International Review, 42, 139-152.

Saks, A. M. (1995). Longitudinal field investigation of the moderating and mediating effects of self-efficacy on the relationship between training and newcomer adjustment. Journal of Applied Psychology, $80,211-225$.

Schmidt, R. A., \& Björk, R. A. (1992). New conceptualizations of practice: Common principles in three paradigms suggest new concepts for training. Psychological Science, 3, 207-217.

Shebilske, W. L., Goettl, B. P., Corrington, K., \& Day, E. A. (1999). Inter-lesson spacing and task-related processing during complex 
skill acquisition. Journal of Experimental Psychology: Applied, 5, 413-437.

Shebilske, W. L., Goettl, B. P., \& Regian, J. W. (1999). Individual and group protocols for training complex skills in laboratory and applied settings. In D. Gopher \& A. Koriat (Eds.), Attention and performance XVII: Cognitive regulation of performance: Interaction of theory and application (pp. 401-432). Hillsdale, NJ: Erlbaum.

Shebilske, W. L., Jordan, J. A., Goettl, B. P., \& Paulus, L. E. (1998). Observation versus hands-on practice of complex skills in dyadic, triadic, and tetradic training teams. Human Factors, 40, 525-540.

Shebilske, W. L., Regian, J. W., Arthur, W., Jr., \& Jordan, J. A. (1992). A dyadic protocol for training complex skills. Human Factors, 34, 369-374.

Slavin, R. E. (1983). When does cooperative learning increase student achievement? Psychological Bulletin, 94, 429-445.

Stajkovic, A. D., \& Luthans, F. (1998). Self-efficacy and work-related performance: A meta-analysis. Psychological Bulletin, 124, 240-261.

Stanne, M. B., Johnson, D. W., \& Johnson, R. T. (1999). Does competition enhance or inhibit motor performance: A meta-analysis. Psychological Bulletin, 125, 133-154.

Taylor, P. J., Russ-Eft, D. F., \& Chan, D. W. L. (2005). A meta-analytic review of behavior modeling training. Journal of Applied Psychology, 90, 692-709.

Tudge, J. R. (1992). Processes and consequences of peer collaboration: A Vygotskian analysis. Child Development, 63, 1364-1379.

Vygotsky, L. S. (1978). Mind in society: The development of higher psychological processes. Cambridge, MA: Harvard University Press.

Wentzel, K. R. (2002). Peer relationships and collaborative learning as contexts for academic enablers. Social Psychology Review, 31, 366-377.

Wood, R. E., \& Bandura, A. (1989). Social cognitive theory of organizational management. Academy of Management Review, 14, $361-384$.
Eric Anthony Day is an associate professor in the Department of Psychology at the University of Oklahoma. He received his Ph.D. in psychology from Texas A\&M University in 1998.

Paul R. Boatman is a doctoral candidate in the Department of Psychology at the University of Oklahoma, where he received an M.S. in psychology in 2004.

Vanessa Kowollik is a doctoral candidate in the Department of Psychology at the University of Oklahoma, where she received an M.S. in psychology in 2005.

Jazmine Espejo is a consultant with Development Dimensions International, Pittsburgh, Pennsylvania. She received her $\mathrm{Ph} . \mathrm{D}$. in psychology from the University of Oklahoma in 2006.

Lauren E. McEntire is a doctoral candidate in the Department of Psychology at the University of Oklahoma, where she received an M.S. in psychology in 2004.

Rachel E. Sherwin is a book representative with Thomson Wadsworth, Norman, Oklahoma. She received her B.S. in psychology from the University of Oklahoma in 2005 .

Date received: September 25, 2006

Date accepted: May 9, 2007 